\title{
Paying attention to biased attention in drug addiction
}

\author{
Karen D. Ersche*
}

Department of Psychiatry, and Behavioural and Clinical Neuroscience Institute, University of Cambridge, Cambridge, UK

It is well recognized that individuals who are dependent on addictive drugs exert a bias in selective attention toward drug-related stimuli, ${ }^{1}$ which can influence their craving for the drug and their drug-taking behavior. Over the past decade, this phenomenon of attentional bias has grabbed the attention of many researchers in the field of addiction.

The observation that disruptions in cognitive processes are linked to an emotional state (for example, craving), which in turn is known to trigger relapse, fueled hopes that attentional bias might be useful in clinical practice when predicting the risk of relapse in patients leaving treatment. It was also hoped that the attenuation of attentional bias, either though training or pharmacotherapy, might help reduce experiences of drug cravings and thereby the risk of relapse.

Consequently, over the past decade, much research has been concerned with elucidating the cognitive mechanisms that underlie this processing bias and its relationship to the risk of relapse. This special issue brings together four articles that highlight the progress of attentional bias research in this area, especially with regard to the pharmacological and neural correlates of drug addiction and their relevance to clinical practice.

The contribution by Field et al. provide an overview of the theoretical context of the phenomenon and the most common ways of measuring it. In critically reviewing the evidence for attentional bias predicting relapse, they call into question the usefulness of attentional bias as a general means of estimating the continuing risk of relapse following discharge from treatment. Their main point is that attentional bias alters in response to motivational states and may therefore not serve as a robust predictor of the risk of relapse over time, for example in several weeks' or months' time. The authors, however, are more optimistic about the usefulness of attentional bias in clinical settings if the instability of attentional bias is taken into account. In fact, attentional bias seems to be a

*Address for correspondence: Dr. Karen Ersche, Department of Psychiatry, University of Cambridge, Herchel Smith Building for Brain \& Mind Sciences, Cambridge Biomedical Campus, Cambridge CB2 0SZ, UK.

(Email: ke220@cam.ac.uk) reasonable predictor for immediate drug use, and they posit that new technologies, such as the use of smart phones, might offer new opportunities for the training of drug users in attenuating attentional bias in certain situations. However, the authors do caution that it is too early to judge any relevance for treatment, as the evidence for reducing attentional bias and subsequent drug-taking through cognitive training is still insufficient.

In the second, and related, article in this issue by Hester and Luijten, the focus is on the importance of understanding neural substrates when considering attentional bias as a target for addiction treatment. While these authors also recognize that behavioral measures of attentional bias have been inconsistent in predicting relapse, they argue that identifying the key neural correlates involved in attentional bias may prove to be a much more reliable predictor of treatment outcome than current strategies. As such, they call for neuroimaging research to play a more important role in better understanding the neural networks that underpin this phenomenon. More specifically, they advise that new research should focus on how these networks contribute to the successful avoidance or failure of control over attentional bias - knowledge that might be useful in designing optimal treatment for drug-dependent individuals in the future.

The contribution by Luijten et al evaluates the putative pharmacological mechanisms that underlie attentional bias by reviewing the contemporary literature of experimental medicine studies aimed at ameliorating attentional bias in drug-dependent individuals. Although dopamine antagonists have provided the most promising results so far, this knowledge has not yet been translated into better treatments for drug-dependent individuals. The authors point out the conspicuous lack of studies using non-dopaminergic compounds to investigate attentional bias and the obvious need to apply current research to a more clinically relevant context so that current knowledge can be more readily applied to treatment settings, or implemented in clinical trials.

The final contribution is by Smith et al, who speculate on the usefulness of attentional bias as a diagnostic tool in disentangling recreational drug users 
from those who become dependent substance users. The idea of using the well-established drug-word Stroop paradigm as a complementary, objective measure of attentional bias, in addition to self-reports of drug usage, is interesting, especially given that drug-taking histories are generally underreported.

This special issue highlights the progress that attentional bias research has made over the past few years. Crucially, all of the authors in this volume agree on the importance and relevance of this phenomenon to drug addiction, while at the same time cautioning against its current use in clinical practice. It is clear that the potential application of attentional bias is by no means exhausted. Instead, the authors point out how advances made using neuroimaging technology and pharmacological modulation still have much to offer this area of research, and that attentional bias still has potential utility in clinical practice and diagnostic applications.

\section{Disclosure}

The author has no conflicts of interest.

\section{REFERENCE:}

1. Field M, Cox WM. Attentional bias in addictive behaviors: a review of its development, causes, and consequences. Drug Alcohol Depend. 2008; 97(1-2): 1-20. 